\title{
A THEOREM ON FUNCTION SPACES
}

\author{
JAN BAARS, JOOST DE GROOT AND JAN VAN MILL
}

(Communicated by Dennis K. Burke)

\begin{abstract}
Let $X$ and $Y$ be normal and first countable spaces, such that $C_{p}(X)$ and $C_{p}(Y)$ are linearly homeomorphic. Suppose $X^{(\kappa)}$ is countably compact for some $\alpha<\omega_{1}$. We prove that if $\alpha=1$ then $Y^{(\alpha)}$ is also countably compact. The first countability condition in this result is essential. We also present examples that if $\alpha$ is not a prime component, then $Y^{(\alpha)}$ need not to be countably compact.
\end{abstract}

\section{INTRODUCTION}

Let $X$ and $Y$ be Tychonov spaces. By $C(X)$ we denote the set of all realvalued continuous functions on $X$. We endow $C(X)$ with a topological vectorspace-structure by considering it to be a subspace of $\mathbb{R}^{X}$. With this topology we denote $C(X)$ by $C_{p}(X)$.

In [1] Arhangelskii proved that if $C_{p}(X)$ is linearly homeomorphic to $C_{p}(Y)$, and $X$ is compact, then $Y$ is compact. In addition, if $X$ is pseudocompact then $Y$ is pseudocompact. This means in particular that if $X$ and $Y$ are normal then $X$ is countably compact if and only if $Y$ is countably compact. In this note we prove that if $X$ and $Y$ are both normal and first countable such that $C_{p}(X)$ is linearly homeomorphic to $C_{p}(Y)$, then $X^{(1)}$ is countably compact if and only if $Y^{(1)}$ is countably compact $\left(X^{(1)}\right.$ is the set of accumulation points of $X$ ). Our technique is inspired by Arhangelskii [1] and Baars, de Groot, van Mill and Pelant [3]. We give two examples showing that our result is "best possible". There exist a first countable normal space $X$ and a normal space $Y$ such that $C_{p}(X)$ and $C_{p}(Y)$ are linearly homeomorphic but $X^{(1)}$ is not countably compact and $Y^{(1)}$ is countably compact. In addition, there exist two metric spaces $X$ and $Y$ such that $C_{p}(X)$ and $C_{p}(Y)$ are linearly homeomorphic but $X^{(2)}$ is compact while $Y^{(2)}$ is not compact $\left(X^{(2)}\right.$ is the second derivative of $X$ ).

Received by the editors April 28, 1988.

1980 Mathematics Subject Classification (1985 Revision). Primary 54C35, 57N20.

Key words and phrases. Function spaces. 


\section{Preliminaries}

In this section we give some results from Baars and de Groot [2], and results and definitions from Arhangelskii [1], which we use in section 2.

Let $X$ be a toplogical space and $A$ a subset of $X$. Let $Y=Y_{X, A}$ be the quotientspace obtained from $X$ by identifying $A$ to one point, say $\infty$. Let $C_{p, A}(X)$ be the subspace of $C_{p}(X)$ consisting of those functions which vanish on $A$, and let $C_{p, 0}(Y)$ be the subspace of $C_{p}(Y)$ consisting of those functions which are zero at $\infty$.

If two linear spaces $X$ and $Y$ are linearly homeomorphic then we denote that by $X \sim Y$.

1.1 Lemma [2]. Let $X$ be a space and $A$ a subset of $X$. Then $C_{p, A}(X) \sim$ $C_{p, 0}(Y)$.

For a topological space $X$ we define for every ordinal $\alpha$ the $\alpha$-th derivative $X^{(\kappa)}$ by transfinite induction as follows: (see [5])

(a) $X^{(0)}=X$ and $X^{(1)}=\{x \in X \mid x$ is an accumulation point of $X\}$.

(b) If $\alpha$ is a successor, say $\alpha=\beta+1$, then $X^{(\alpha)}=\left(X^{\beta)}\right)^{(1)}$.

(c) If $\alpha$ is a limit ordinal then $X^{(\alpha)}=\bigcap_{\beta<\alpha} X^{(\beta)}$.

An ordinal $\alpha$ is a prime component whenever for all ordinals $\beta$ and $\delta$ with $\alpha=\beta+\delta$ we have $\delta=0$ or $\delta=\alpha$. For every ordinal $\alpha$ denote by $\alpha^{\prime}$ the largest prime component which is less than or equal to $\alpha$.

By $C_{p, 0}([1, \alpha])$ we mean the subspace of $C_{p}([1, \alpha])$ consisting of those functions which are zero at $\alpha$.

The next lemma and theorem can be found in [2].

1.2 Lemma. Let $\alpha$ be an ordinal. Then $C_{p, 0}([1, \alpha]) \sim C_{p}([1, \alpha])$.

1.3 Theorem. Let $\omega \leqq \alpha, \beta<\omega_{1}$. Then $C_{p}([1, \alpha]) \sim C_{p}([1, \beta])$ iff $\alpha \leqq$ $\beta<\alpha^{\omega}$.

The following definitions can be found in [1]. Let $X$ and $Y$ be Tychonov spaces, and $\phi: C(X) \rightarrow C(Y)$ a linear mapping. For every $y \in Y$, the support of $y$ in $X$ is defined to be the set $\operatorname{supp}(y)$ of all $x \in X$ satisfying the condition that for every neighborhood $U$ of $x$, there is an $f \in C(X)$ such that $f(X \backslash U)=\{0\}$ and $\phi(f)(y) \neq 0$. For a subset $A$ of $Y$, we denote $\bigcup_{y \in A} \operatorname{supp}(y)$ by $\operatorname{supp} A$. Furthermore $\phi$ is said to be effective if for every $f, g \in C(X)$ and $y \in Y$, such that $f$ and $g$ coincide on a neighborhood of $\operatorname{supp}(y), \phi(f)(y)=\phi(g)(y)$.

A subset $A$ of $X$ is said to be bounded if for every $f \in C(X), f(A)$ is bounded in $\mathbf{R}$.

1.4 Proposition. ([1] Arhangelskii). Let $X$ and $Y$ be Tychonov spaces and $\phi: C_{p}(X) \rightarrow C_{p}(Y)$ a linear homeomorphism. Then

(a) $\phi$ is effective, 
(b) if $A$ is a bounded subset of $Y$, then $\operatorname{supp} A$ is bounded in $X$.

For details about ordinals we refer to [5] and [6].

\section{FUNCTION SPACES}

In this section we prove the results, announced in the Introduction.

2.1 Lemma. Let $X$ and $Y$ be Tychonov spaces and $\phi: C_{p}(X) \rightarrow C_{p}(Y)$ a homeomorphism. Suppose that $\left(f_{n}\right)_{n \in N}$ is a sequence in $C_{p}(X)$ such that $f_{n}$ converges pointwise to a discontinuous function $f \in \mathbb{R}^{X}$. Suppose $g: Y \rightarrow \mathbb{R}$ is an accumulation point of the set $\left\{\phi\left(f_{n}\right) \mid n \in \mathbb{N}\right\}$. Then $g$ is not continuous.

Proof. Since $\left\{f_{n} \mid n \in \mathbb{N}\right\}$ is closed and discrete in $C_{p}(X)$ we have $\left\{\phi\left(f_{n}\right) \mid\right.$ $n \in \mathbb{N}\}$ is closed and discrete in $C_{p}(Y)$.

2.2 Theorem. Let $X$ and $Y$ be topological spaces which are both normal and first countable and let $C_{p}(X)$ and $C_{p}(Y)$ be linearly homeomorphic. Then $X^{(1)}$ is countably compact if and only if $Y^{(1)}$ is countably compact.

Proof. Suppose $X^{(1)}$ is not countably compact and $Y^{(1)}$ is countably compact. Since $X^{(1)}$ is not sequentially compact, there exists a closed discrete set $F=$ $\left\{x_{n} \mid n \in \mathbb{N}\right\}$ in $X^{(1)}$. For every $n \in \mathbb{N}$ let $\left\{U_{j}^{n} \mid j \in \mathbb{N}\right\}$ be a decreasing open base at $x_{n}$ and $f_{j}^{n}$ a Urysohn function such that $f_{j}^{n}\left(x_{n}\right)=1$ and $f_{j}^{n}\left(X \backslash U_{j}^{n}\right)=$ 0 . Then $f_{j}^{n} \rightarrow \chi_{x_{n}}$ pointwise, where $\chi_{x_{n}}$ is the characteristic function of $x_{n}$. Notice that $\chi_{x_{n}}$ is discontinuous. Furthermore let $\phi: C_{p}(X) \rightarrow C_{p}(Y)$ be a linear homeomorphism and let $g_{j}^{n}=\phi\left(f_{j}^{n}\right)$.

Claim. For every $y \in Y$ and $n \in \mathbb{N}$, the set $\left\{g_{j}^{n}(y) \mid j \in \mathbb{N}\right\}$ is bounded in $\mathbf{R}$.

Suppose not. Then there are $y \in Y$ and $n \in \mathbb{N}$, such that without loss of generality for every $k \in \mathbb{N}$ there is $j_{k} \in \mathbb{N}$, with $g_{j_{k}}^{n}(y) \geqq 2^{k}$. The function $f=\sum_{k=1}^{\infty} 2^{-k} f_{j_{k}}^{n} \in C_{p}(X)$, so $\phi(f)=\sum_{k=1}^{\infty} 2^{-k} g_{j_{k}}^{n} \in C_{p}(Y)$. But then we have a contradiction since $\phi(f)(y)=\sum_{k=1}^{\infty} 2^{-k} g_{j_{k}}^{n}(y)=\infty$.

For every $y \in Y$, let $A_{y}$ be compact in $\mathbb{R}$ such that $\left\{g_{j}^{n}(y) \mid j \in \mathbb{N}\right\} \subset A_{y}$. Then $\prod_{y \in Y} A_{y}$ is a compact subset of $\mathbb{R}^{Y}$. Since $\left\{g_{j}^{n} \mid j \in \mathbb{N}\right\} \subset \prod_{y \in Y} A_{y}$, $\left\{g_{j}^{n} \mid j \in \mathbb{N}\right\}$ has an accumulation point $\sigma_{n}$. By Lemma 2.1, $\sigma_{n}$ is discontinuous, say at $y_{n}$. Notice that $y_{n} \in Y^{(1)}$. Since $Y^{(1)}$ is sequentially compact, without loss of generality we may assume that there is $y \in Y$ such that $y_{n} \rightarrow y$. Let $\left\{V_{n} \mid n \in \mathbb{N}\right\}$ be a decreasing open base at $y$. Without loss of generality $y_{n} \in V_{n}$.

Since $Y$ is first countable, for every $n \in \mathbb{N}$ there is a sequence $\left(y_{k}^{n}\right)_{k}$ in $V_{n}$ such that $y_{k}^{n} \rightarrow y_{n}$ and

$$
\sigma_{n}\left(y_{k}^{n}\right) \nrightarrow \sigma_{n}\left(y_{n}\right) .
$$

Let $K=\bigcup_{n \in \mathbb{N}} \bigcup_{k \in \mathbb{N}}\left\{y_{n}, y_{k}^{n}\right\} \cup\{y\}$. Then $K$ is compact. Indeed, let $\mathscr{V}$ be an 
open cover of $K$. There is $V \in \mathscr{V}$ with $y \in V$. There is $n_{0} \in \mathbb{N}$ such that $y \in V_{n_{0}} \subset V$. Then $\bigcup_{n \geqq n_{0}} \bigcup_{k \in N}\left\{y_{n}, y_{k}^{n}\right\} \cup\{y\} \subset V$. Since $\bigcup_{n<n_{0}} \bigcup_{k \in N}\left\{y_{n}, y_{k}^{n}\right\}$ is compact, we are done.

Since $K$ is compact, it is bounded in $Y$. So by Proposition 1.4, $\overline{\operatorname{supp} K}$ is bounded in $X$. Since $F$ is closed and discrete and $X$ is normal, $F$ is not bounded. This implies that there is $n \in \mathbb{N}$ such that $x_{n} \notin \overline{\operatorname{supp} K}$. Since $X$ is regular there is $j_{0} \in \mathbb{N}$ and a neighborhood $V$ of $\overline{\operatorname{supp} K}$ such that $U_{j_{0}}^{n} \cap V=\varnothing$. So for every $z \in K$ and $j \geqq j_{0}, f_{j}^{n}$ and the zero function on $X$ are equal on $V$, which is a neighborhood of $\operatorname{supp}(z)$. Since $\phi$ is linear and effective, this implies that $g_{j}^{n}(z)=0$ for every $j \geqq j_{0}$ and $z \in K$. But then $\sigma_{n}\left(y_{k}^{n}\right)=0$ and $\sigma_{n}\left(y_{n}\right)=0$, which gives a contradiction with $(*)$.

By $X \oplus Y$ or $\oplus_{i=1}^{\infty} X_{i}$ we denote the topological sum of the topological spaces $X$ and $Y$ or $X_{i}(i \in \mathbb{N})$, respectively.

2.3 Example. In this example we show that the first countability condition in Theorem 2.2 is essential.

Let $X=\oplus_{i=1}^{\infty}[1, \omega]_{i}$. Let $A=X^{(1)}$ and $Y=Y_{X, A}$ the quotient space obtained from $X$ by identifying $A$ to single point, say $\infty$. Then $X$ is clearly first countable and normal, and $Y$ is normal but not first countable. By Lemma 1.1 we have $C_{p, A}(X) \sim C_{p, 0}(Y)$. Furthermore we have

$$
\begin{aligned}
C_{p, A}(X) & \sim \prod_{i=1}^{\infty} C_{p, 0}([1, \omega])_{i} \\
& \sim \prod_{i=1}^{\infty} C_{p}([1, \omega]) \quad(\text { Lemma 1.2a }) \\
& \sim C_{p}(X) .
\end{aligned}
$$

Notice that for every Tychonov space $Z$ and for every $z \in Z, C_{p}(Z) \sim$ $C_{p, 0}(Z) \times \mathbb{R}$, where $C_{p, 0}(Z)$ consists of those functions in $C_{p}(Z)$ which vanish at $z$. So by Lemma $1.2, C_{p}([1, \omega]) \sim C_{p}([1, \omega]) \times \mathbb{R}$. This implies $C_{p}(X) \sim$ $C_{p}(X) \times \mathbb{R}$. So

$$
\begin{aligned}
C_{p}(X) & \sim C_{p}(X) \times \mathbb{R} \\
& \sim C_{p, A}(X) \times \mathbb{R} \\
& \sim C_{p, 0}(Y) \times \mathbf{R} \\
& \sim C_{p}(Y) .
\end{aligned}
$$

However $X^{(1)}=A$ is not countably compact, and $Y^{(1)}=\{\infty\}$ is countably compact.

From Theorem 2.2 and the result in [1] for normal spaces, that if $C_{p}(X)$ and $C_{p}(Y)$ are linearly homeomorphic and $X$ is countably compact, then $Y$ is countably compact, one could conjecture the following: Let $\alpha$ be an arbitary ordinal. If $X$ and $Y$ are both normal and first countable spaces such that 
$C_{p}(X)$ and $C_{p}(Y)$ are linearly homeomorphic and $X^{(\alpha)}$ is countably compact, then $Y^{(\alpha)}$ is countably compact.

In the next example we show that if $\alpha$ is not a prime component, then the conjecture is false.

2.4 Example. Let $\alpha<\omega_{1}$ be an ordinal which is not a prime component. Observe that in this situation $1 \leqq \alpha^{\prime}<\alpha$.

Let $X=\oplus_{i=1}^{\infty}\left[1, \omega^{\alpha^{\prime}}\right]_{i}$ and $Y=\oplus_{i=1}^{\infty}\left[1, \omega^{\alpha}\right]_{i}$. By Theorem 1.3, $C_{p}\left[1, \omega^{\alpha^{\prime}}\right]$ $\sim C_{p}\left[1, \omega^{\alpha}\right]$, so that $C_{p}(X) \sim C_{p}(Y)$. But $Y^{(\alpha)} \approx \mathbb{N}$ (see [2] or [6] p. 155) which is not countably compact, and $X^{(\alpha)}=\varnothing$ which is countably compact.

Questions. (1) Is the above conjecture true for prime components?

(2) Does Theorem 2.2 still hold if normal is replaced by Tychonov?

\section{REFERENCES}

1. A. V. Arhangelskii, On linear homeomorphisms of function spaces, Soviet Math. Dokl. 25 (1982), 852-855.

2. J. Baars and J. de Groot, An isomorphical classification of function spaces of zero dimensional locally compact separable metric spaces, Comm. Math. Univ. Corolinae 29, 3 (1988), 577-595..

3. J. Baars, J. de Groot, J. van Mill and J. Pelant, On topological and linear homeomorphisms of certain function spaces (to appear in top. and appl.).

4. K. Kuratowski and A. Mostowski, Set theory, North-Holland Publishing Company, 1976.

5. Z. Semadeni, Banach spaces of continuous functions, PWN Warszawa, 1971.

6. W. Sierpiǹski, Cardinal and ordinal numbers, PWN Warszawa, 1958.

FACUlteit Wiskunde en INFORMatica, Universiteit VAN AMSTERdam, RoEtersstraAt 15, 1018 WB AMSTERDAM, THE NETHERLANDS

Faculteit Wiskunde en INformatica, VRije Universiteit, De BoelelaAN 1081, $1081 \mathrm{HV}$ AMSTERDAM, The NetherLaNDS 